\title{
Chronic Poverty: The Implications of Bullying, Trauma, and the Education of the Poverty-Stricken Population
}

\author{
Kevin Johnson, M.Ed. \\ Liberty University, United States
}

Doi: 10.19044/ejes.s.v6a6

URL:http://dx.doi.org/10.19044/ejes.s.v6a6

\begin{abstract}
Chronic poverty is a worldwide epidemic, and communities must take a proactive approach to assist the poor by extending a hand to lift them up and not hold them down. Tribulations are part of life, but are some afflictions selfimposed, escalated, or reinforced by living in deprived contextual environments. Poverty-stricken people experience more trauma throughout their lifetime; they are less educated than their counterpart, causing them to become targets in school, increasing their chances of being bullied and demoralized. Bullying is not a rite of passage, and it has lifelong effects that reveal itself in adulthood by strengthening generational curses, oppressing families and communities, expanding the educational gap, and reinforcing the cycle of chronic poverty. The research depicted in this article explores the correlation between poverty, human development, trauma, pedagogical implications, and bullying, characterizing the detrimental ramifications in adulthood. The paper analyzes bully symptomology, the etiology of traumatic experiences, and how the consequences of chronic poverty affect human development that expands the educational gap between minorities and white students. Trauma-focused cognitive behavioral therapy is an effective empirically-based treatment modality to combat the symptoms of posttraumatic stress. School systems must do a better job of educating traumatized children living in poverty. The research ventures to explain chronic poverty's role in human development, traumatology, and education, taking an inclusive approach to providing solutions to create a cultural shift that will change the contextual environment and propel people to become selfsufficient, more educated, and equipped to break the generational curse of chronic poverty.
\end{abstract}

Keywords: Chronic poverty, trauma, bullying, CPTSD, education. 


\section{Introduction:}

President Thomas Jefferson wrote, "We hold these truths to be selfevident, that all men are created equal, that they are endowed by their Creator with certain unalienable Rights, that among these are life, liberty and the pursuit of happiness." In the eyes of their Creator, all men are created equal in the image of God, but throughout history, a man divided equality and justice for all during slavery, Jim Crow, and the Civil Rights Movement. Many people are miseducated and do not comprehend that the Civil Rights Movement was not just for the Black race but all people, nationalities, ethnicities, gender, and the disabled; the fight was for fairness for every color and creed. During this period, the mentally challenged and disabled had struggles and battles of their own. Fortunate enough, there were advocate groups and parents who were willing to stand up and fight for the equal rights of those who could not stand and fight for themselves. The mentally challenged and disabled have been marginalized from society and the classrooms for years. Is it possible for people who are reared in chronic poverty to beat the odds and succeed when all odds are stacked against them? How is it possible to provide quality education and security when children living in poverty normally stand out from the crowd due to hunger, lack of resources, and poor hygiene, not adequately having clothing, shelter, and other basic needs?

In President John F. Kennedy's inaugural address, he proclaimed, "And so, my fellow Americans: ask not what your country can do for you — ask what you can do for your country." What happens when the country placed certain people at a significant disadvantage by being born the wrong color? How can those who live a life of chronic poverty and generational curses of destitute do anything for their country when they lack education and financial resources? Chronic poverty orchestrates a life of injustice, discrimination, prejudice, and unequal opportunities, regardless of the misconception that people are created equal. Chronic poverty puts people at higher risk of poor mental and physical health, risky behaviors, substance use disorders, complex posttraumatic stress, and live a traumatic and detrimental lifestyle that stifles education, religious beliefs, maturity, and personal growth, which has the propensity to bind individuals mentally, spiritually, and emotionally. Lifetime adversities and trauma correlate with low self-esteem, mental illness, lower socioeconomic status, and small educational achievement, especially in the Black and Latino populations who happen to be underrepresented to most studies. Myers, Wyatt, Ullman, Loeb, Chin, Prause, Zhang, Williams, Slavich, and Liu (2015) asserted that minorities experience unique stressors that impact their health including stressors such as neglect by society, discrimination, and multiple traumatic experiences. Hardships in life devitalize people, resulting in pain, suffering, and stress, while others who perceived the same traumatization rally up the power to not only survive but thrive. 
Generational poverty poses a distinct predisposition to the etiology of the essence of traumatization because poverty can be a "little leaven" that levels an entire generation. As the root cause of many traumatic events, poverty has the proclivity to construct a unique dynamic that makes trauma challenging to isolate and identify as a tragedy because families are described as dysfunctional. Lowdermilk and Brunache (2013) professed that many children reared in poverty mirrors the same behaviors as parents and their community. They reflect those individuals growing up in demanding environments, reporting negative/absent parent, parents with multiple sexual partners or engaged in prostitution, substance use disorder, violence in the home and community, verbal/physical abuse, unsanitary conditions, lack of finances and resources, and parents demonstrate very low educational expectations for their children, and all of this happens within the children's nuclear family. With the dismantling of the family structure, is it possible to close the achievement gap and break the generational curse of the chronicity of poverty?

\section{Chronic Poverty and Human Development}

Researchers examine the psychological and emotional development of children living in poverty based on parental guidance and the methods parents use to rear their children. Evans and Kim (2012) reported that "in the last two decades, many scholars have investigated the underlying psychological processes that explain why childhood poverty has such pervasive ill effects on human development" (p. 43). Evidence substantiates that poverty causes chronic stress, which deteriorates human functioning in every faculty of the body, especially memory, brain development, and emotional regulation. Children in poverty face unusual stressors that children living in middle-class do not know about because they live in a contextual environment perceived as safe and nurturing. Amatea and West (2007) declared that children living in poverty are considerably more likely than children from the middle-class to report increased levels of anxiety and depression, exhibiting a higher frequency of behavioral and academic difficulties, and a lower level of positive academic interaction and engagement in the school system. Collins, Connors, Donohue, Gardner, Goldblatt, Hayward, Kiser, Strieder, and Thompson (2010) reported that children who grow up in urban poverty display symptoms of complex posttraumatic stress disorder; "complex trauma is a varied and multifaceted phenomenon, frequently embedded in a matrix of other psychosocial problems (e.g., neglect, marital discord, and domestic violence that carry ongoing threat)" (p. 12). As reported by Gabrielli, Gill, Koester, and Borntrager (2013), it takes a thorough understanding of the ramifications of trauma and the unfolding developmental processes of childhood as it is experienced in a particular culture, without neglecting to 
understand the nature of historical trauma, as aggregated spiritual, physical, emotional, and psychological wounding over the lifespan and across generations.

Studies show that the ability to sense touch occurs around eight weeks of gestation, and fetal development of the other sensory organs begin around five weeks of conception and continue to refine through birth and early childhood (Uhernik, 2017). While in utero, the fetus can hear and respond to sounds and noises outside of the womb. Many parents play music, read books, and talk to their fetuses throughout the pregnancy, interacting by touching certain spots of the mother's belly by poking or grabbing a hand or foot when it is visible. At birth, the newborn can only see eight to ten inches, and research denotes this short distance is "precisely the range required for gazing into the caregiver's eyes when held and for beginning the visual and interactive dance of attachment and bonding" (Uhernik, 2017, p. 54). Establishing a bond and attachment between the mother and the baby is the first step in psychosocial development, which is vital for successful progression throughout life. As reported by Kim, Fonagy, Allen, and Strathearn (2014),

Maternal sensitivity to infant distress and non-distress as predictors of infant-mother attachment security. Maternal attachment trauma, particularly when unresolved, presents to the mother's attunement to and management of her infant's distress. Although this has not yet been the subject of direct empirical scrutiny, attachment researchers have long speculated that infants' distress signals may activate unresolved traumatic memories in their respective mothers, thereby initiating a cascade of compromised maternal responses. (p. 354)

The maternal reactions manifest traumatic memories for the mother and can affect memory and cognitive abilities for the newborn.

Erik Erikson was a renowned psychologist and anthropologist who identified eight stages of psychosocial stages of development, from birth to old age. Everyone must discover her sense of regulation as she interacts with the environment and the biological, emotional, and psychological idiosyncrasies in life. Infants must establish trust so that they feel safe in knowing that their needs will be met, and they will not be neglected. Stability and a sense of security allow infants to see the world as secure and a dependable place, encouraging optimism about the future and having confidence in themselves and other people. Mounting research postulates that fetuses can learn and have short term memory, which is believed to help the fetus bond with his or her mother. When researchers used vibroacoustic stimulation, Gonzalez-Gonzalez, Suarez, Perez-Pinero, Armas, Domenech, and Bartha (2006) asserted that newborns in utero recognized the stimulus. Newborns habituated sooner than babies who were not stimulated before birth. This evidence suggests that babies encouraged during fetal life were able to 
learn, recall, and memorize. Fetuses demonstrate advancement in cognitive abilities throughout the fetal development period because the brain is this amazing organ that has the potential for plasticity, neurogenesis, and the creation of new neural pathways. Researchers like Otto Rank and Wilfred Bion hypothesized that being born is a traumatizing experience because the brain becomes bombarded by outside stimuli, causing the infant to experience chaos and traumatization.

The hypothesis is not too far-fetched, considering that research postulated that learning and memory formation occur in utero. Memory and cognitive abilities learned inside the womb clash with the new environment when the infant is removed from the womb. The baby takes in an array of sounds, light, and different touches and smells that were limited in utero. If the trauma of being born is significant, it is the caregiver's responsibility to ensure that a secure attachment is formed, because this lessens the "traumatic experience" by providing a contextual environment where the infant can establish trust instead of mistrust. The strength of the mother's attachment, mentally and physically, predicates the extensiveness of the trauma or determines if the traumatic birthing experience will be lessened and managed by providing love and trust instead of neglect and mistrust. Aside from the possible functions of recognition and attachment to the mother, the significance of fetal memory and cognition are necessary for the promotion of breastfeeding and language acquisition (James, 2010).

Lipina, Colombo, and Jorge (2010) postulated that SES levels are associated with a degree of hemispheric specialization and gray/white matter volumes, concluding that reduced language skills were associated with lower SES and related to less underlying neuronal specialization. According to James (2010), "A large amount of evidence from habituation, classical conditioning and exposure learning in humans substantiate that the fetus can learn; however, there is no sound evidence showing that extra auditory stimulation is of benefit to child development" (p. 52). Chronic poverty adds a different variable to how newborns learn; the implications poverty has on babies as they develop through the various stages of life impact their education, increase stress levels, exposure to violence, and put them at a higher risk of being traumatized.

Mounting studies show the detrimental impact of poverty on brain development in children and adolescents, revealing how and why there is an achievement gap in education between Blacks, Latinos, and their white counterpart. Cortisol is the stress hormone needed when there is distress and the fight and flight response catapults into high gear when the body senses imminent danger or significant stress. High levels of cortisol affect the amygdala that regulates emotions, and it can cause coronary heart disease, hypertension, and high cholesterol levels. Children living in poverty have 
higher levels of cortisol and other stress markers that have disadvantageous effects on emotional intelligence, regulation, academics, and executive functioning. According to Blair and Raver (2016),

Executive function is essential for self-regulation and school readiness and is a fundamental building block of early cognitive and social competence. Available evidence indicates that the effects of socioeconomic and early psychosocial disadvantage on cortisol and brain structure partially mediate effects of poverty on the development of executive function in childhood. Impact of poverty on brain development and executive function are likely one critical pathway, along with reduced stimulation for learning, through which poverty is associated with gaps in school readiness and achievement and positive life outcomes. (p. 4)

Taylor and Barrett (2018) professed that "the impact of developmental trauma in the early years can have a disproportionate effect on the growth, day-to-day functioning and life chances of a young person" (p.73).

Other studies have shown how nonhumans' neural pathways evolve, creating new and advanced channels when placed in environments where they were stimulated and challenged to interact with devices and manipulatives that promoted higher levels of cognitive functioning. According to Lipina and Colombo (2009), elements of executive function, memory, and selfmonitoring tend to be lower in children who live in poverty. The researchers used basic tasks to "assess skills related to dorsolateral prefrontal (working memory), anterior cingulate (cognitive control), and ventromedial (reward processing) prefrontal systems, the results showed a consistent disparity between children from lower and middle SES groups" (p. 583). As various studies report, poverty-stricken children are placed at a disadvantage from birth throughout the different stages of life. If preventive measures, interventions, and programs are not tailored to meet this demographic, the cycle of generational poverty will continue, creating a new environmental DNA passed down to its offspring. How do society and educational systems close the divide in education, learning, and development? According to Ladd (2012), "Addressing the educational challenges faced by children from disadvantaged families will require a broader and bolder approach to education policy than the recent efforts to reform schools" (p. 204).

\section{Chronic Poverty and Trauma}

The majority of psychological research on the consequences of trauma, poverty, and education has been conducted at the level of the individual, typically studying the persons' trauma histories, and trauma exposures with their symptoms, demographics, and contextual environment (Klest, 2012). As reported by Collins et al. (2010), there is critical evidence suggesting that children, adolescents, and families growing up in poverty are more likely to 
experience multiple traumas, significant adverse life events, and develop convoluted symptoms of traumatic distress at disproportionate rates. The research presented in this literature explores trauma from a specific group that it impacts and consists of a subset or region of individuals, instead of looking at trauma as a single catastrophe but a subset of issues or deficiencies that culminate into traumatic experiences from living a life of generational curses and chronic poverty. It is no secret that Blacks, Latinos, and other minorities constitute most of this vulnerable and underserved group. As reported by Collins et al. (2010), "Efforts to explain the severity and chronicity of reactions to repeated traumas traditionally focus on the cumulative effects of multiple traumatic episodes" (p. 11). Trauma can be experienced or witnessed vicariously, and second-hand trauma can be worse for the person who is helping the survivor or the one who saw the event from a different perspective. After the 911 attack in New York, people suffered from posttraumatic stress as far as Texas and Oklahoma; it is understood as a profoundly disrupting experience that can threaten the well-being and safety of those involved (Gabrielli, Gill, Koester, \& Borntrager, 2013).

Many psychologists and researchers describe trauma differently, characterizing a common thread that ties the theories together, but a definitive definition of trauma is difficult to establish because tragedy can be subjective. A clinician and client can disagree about an episode being traumatic or not, because of the client's apperception, resiliency, and personal growth. Scott and Briere (2015) discussed the ambiguous description of trauma that is characterized by the Diagnostic and Statistical Manual of Disorders, 5th edition (DSM-5), which provides a broad definition with limitations, causing conflicting information in determining if an event satisfies statistical definitions of trauma. Scott and Briere (2015) denoted their interpretation and professed that "an event is traumatic if it is extremely upsetting, at least temporarily overwhelms the individual's internal resources, and produces lasting psychological symptoms" (p. 10). If the definition defined by the DSM5 is adhered to strictly, many clients would not be classified as a trauma survivor.

There is a fallacy that a traumatic event must be a devastating isolated experience that keeps resurfacing in a person's life. Trauma does not have to be a single catastrophe but a culmination of small, insignificant issues that converge together until all the pieces of life's problems connect like the perfect jigsaw puzzle. The mental symptomology of traumatic experiences can debilitate people from being able to access coping skills to keep intrusive thoughts at bay, and they lack the cognitive abilities to manage and process the traumatic experience and the possible manifestation of psychological disorders. It takes a thorough understanding of the ramifications of trauma and the unfolding developmental processes of childhood as it is experienced in a 
particular culture to understand the nature of historical trauma, as aggregated spiritual, physical, emotional, and psychological wounding over the lifespan and across generations (Gabrielli, Gill, Koester, \& Borntrager, 2013). Trauma that affects poverty-stricken cultures covers a broad range of atrocities that destroys the sense of safety within the individuals' community, including neighborhoods, schools, churches, towns, and other places where people conjugate together but separately ("Center for Substance Abuse," 2014).

Studies have identified the problem and determined that increased trauma is associated with poverty and deprivation. The consistent restriction and limited opportunities that characterize poverty can lead to daily hassles and unexpected traumatic events like the lack of food or disconnection from water, electricity, and other supplies (Shamai, 2017). It is normal to experience trauma across the lifespan; for most people, individuals and communities usually respond to tragedy with resilience, because many influences shape the effects of trauma among individuals and families. Due to other factors and circumstances, it is not just the traumatic episode that predicts the outcome, but also the episode's context and the interactions between family support, first responders, counselors, psychological first aid providers, and community leaders ("Center for Substance Abuse," 2014). Severe childhood adversity, in the form of traumas such as physical and sexual abuse, alters young people's transition into adulthood due to social and behavioral reasons, but also due to the physiologic and neurobiological changes that occur due to chronic stressors. Considering the impact and effects of poverty and trauma, Myers et al. (2015) proclaimed that numerous studies refute the results expected because both African Americans and Latinos(as) appeared to be rather resilient, despite their higher than normal stressors and burdens of adversities and traumatizing experiences. It is safe to infer that people who have lived a life of trauma and lack resources all of their lives have become conditioned to become content in whatever state they find themselves in their contextual world. They do not know the hidden rules of the middle-class, so what appears to be poverty and trauma for the middle-class is healthy everyday living for people residing in poverty.

Research substantiates that fifty percent of people will experience trauma in their lifetime. This traumatic experience for only fifty percent of the population is up for debate if we believe in the work of Otto Rank who proclaimed that being born is a traumatic experience, beginning when the infant inhaled his or her first breath. Stewart (2014) purported that Arthur Schopenhauer and Otto Rank discussed suffering because of birth itself, referring to being born as a traumatic experience. Aside from birth, people in poverty are exposed to the daily hassles that cause stress. Klest (2012) reported that models of trauma and coping include the erosion of family processes and dynamics (structure, relations, coping) in the context of community violence 
and poverty, explaining the higher likelihood of family violence in the contextual environment of poverty. The traumatic experience by one family member can create a traumatic stress reaction in other family members through association and proximity to the danger or the catastrophe (Kiser \& Black, 2005).

\section{The Domino Effect: Chronic Poverty, Trauma, and Bullying}

Today, school violence is nothing new, and it continues to become more frequent and deadly in the school systems. Across the globe, educational institutions have active-shooter drills protocols and prepare students, faculty, administrators, and staff what to do when this vicious act of violence occurs. No matter the amount of preparation, no one is ever prepared for the time a school shooting happens in their contextual framework. This type of school violence is evident, reported by newspapers, talk shows, television, social media, and other mediums, repeatedly. As published by Levers (2012), it took the U.S. Department of Education and the U.S. Secret Service to define the parameters of school violence, but one of the most critical findings was "defining school violence as consisting of two sorts: lethal and nonlethal." This research focuses on the nonlethal violence that does not get the attention warranted, and protocols are not protecting the children and ensuring their safety sufficiently. Nonlethal school violence consists of all forms of bullying threats, intimidation, harassment, assault, and sexual assault, constituting physical, verbal, social, and cyberbullying (Levers, 2012). According to Skaine (2015), "bullying is an unwanted, aggressive behavior that involves a real or perceived power imbalance that is repeated, or has the potential to be repeated, over time" (p. 40). Bullied children and adolescents have PTSD, and they need to be treated for the symptoms because traumatized children grow up to become traumatized, broken adults. Herman (2001) reported the significance of abuse in childhood, and how later in life, childhood abuse can contribute to increased risk for cancer, heart disease, stroke, and obesity increasing the risk of alcoholism, depression, suicide attempts, self-harm, and absenteeism from work in adulthood. Bullying is a form of complex posttraumatic stress disorder (CPTSD). Herman (2012) reported that "CPTSD was specifically associated with early-onset and long duration of trauma; it was very rarely found in survivors of natural disasters, and very commonly found in survivors of childhood abuse" (p. 25).

The reoccurrence of the same traumatic experience is what distinguishes CPTSD from PTSD. An individual might have PTSD after a horrific car accident, an isolated sexual assault, or natural disaster, but CPTSD is characterized as having the same traumatic experience repeated on several occasions. Research substantiates that CPTSD symptoms account for the functional impairment of trauma survivors who endured prolonged and 
repeated tragic episodes exceeding the damage attributed to PTSD alone (Herman, 2012). Bullying falls under the category of complex trauma, and it attributes to the psychological effects in adulthood. Children living in poverty can stand out from the crowd because of dirty clothes, holes in shoes, unkempt hair, and poor personal hygiene. Bilic (2015) reported that almost all socioeconomic variables that suggest that a family is poor correlate significantly with victimization, and the results from the same study denoted that $34.8 \%$ of those living in poverty responded violently toward peers because of their more inferior financial status, and $45.7 \%$ were bullied and victimized for being poor. Some bullied children might retaliate by taking extreme violent means of retaliation. In the 1990s, 12 of the 15 school shooting cases that occurred, the shooters had a history of being bullied (Skaine, 2015).

Trauma is an occurrence when an intense experience stuns a child like a bolt out of the blue, overwhelming the child, leaving him or her altered and disconnected from body, mind, and spirit (Steele \& Malchiodi, 2012). Bullying is not harmless; bullying is not child's play; bullying is not a rite of passage. A rite of passage insinuates that bullying is part of life and the school experience, so children should expect to be bullied. Children go to school to learn about history, science, arithmetic, spelling, and using correct grammar, so being bullied is one of the soft skills or indirect learning experiences embedded in the school curriculum. In the academic subjects, students receive a letter grade of pass or fail as they matriculate through school. What grade do students get for enduring four, six, eight, or twelve years of bullying? How do teachers indicate if their students passed or failed? If the student never attempts suicide, is that a passing score?

If the student only has low self-esteem and depression and anxiety, is that considered acceptable to be promoted to another year of punishment? If the individual only has non-suicidal injuries - such as cutting or burning - is that enough for promotion? However, when the person dies by suicide, it is safe to say that he or she failed the rite of passage. Many children believe that when they get older, they will grow up and out of the bullying environment, but the psychological effects and ramifications follow them into adulthood.

Tariq (2011) purported that the problem of most significant concern is the stifling effects produced by the survivor of bullying. Staggering numbers of investigators have asked whether victims of peer aggression experience psychosocial maladjustments, such as depression, anxiety, and low selfesteem, and "it is clearly important to know the answer to this question, so that children's distress does not go unrecognized" (p. 23). Suicide rates have increased tremendously over time in schools, contributing to the fact that those who are being bullied and lack evidence of physical scars, but they suffer away in silence until victimization becomes overbearing, losing hope, and deciding that the only solution for them is to die by suicide (Hendricks \& Tanga, 2019). 
Bullying is not a rite of passage - even though children are steadfast in believing that their trials and tribulations will be over when they become adults. Unfortunately, the research does not predict a happily ever after but a possible lifetime of physical and psychological problems - leaving mental, spiritual, emotional, and physical wounds. Parents and educators must recognize the signs and symptoms of bullying. Often, the physical signs are evident - like unexplained injuries, bruises, broken bones, and the black eye. Bullied children tend to isolate themselves from family and friends, become depressed, and become overly anxious. Vanderbilt and Augustyn (2010) identified several red flags that an individual is being bullied. Some of the more common indications are stomachaches, headaches, insomnia, social problems, lack of friends, and academic failure. It is critical that bullying is considered on an extensive list of differential diagnoses because bullying may overlap with other conditions such as medical illness, learning problems, and psychological disorders (Vanderbilt \& Augustyn, 2010).

Adults who were bullied as a child or adolescent may have a lower socioeconomic status (poverty), engage in risky behaviors, suffer from anxiety and depression, and experience psychosis and have suicidal ideations or attempts. Blad (2016) stated that children should be assessed from birth to understand the extent of bullying on psychosis later in their adult life - with some groups to be nearly five times more likely to suffer from episodes of psychosis by the age of 18. In adulthood, bullied children have higher rates of suicidal thoughts and plans than children who had not been bullied (Wagner, 2016). There is no escape when bullied children grow up to be high school dropouts and experience long-term effects (depression, relationship problems, psychosis, substance use disorder, risky behaviors, and suicide). When the psychological effects of bullying accompany children into adolescent and adulthood, not only did the children fail the rite of passage, but parents failed; educators failed; church leaders failed; the school system failed; society failed these children.

Why is it critical that bullying is discussed trauma and the chronicity of poverty? It is the psychological effects of bullying that warrants the nation's attention if the educational gap is going to be closed. Being bullied as a child or experiencing bullying behaviors have the propensity to continue the cycle of chronic poverty, so the generational curse continues with no end if a cultural shift does not happen soon.

- Children who live in poverty experience higher rates of trauma and bullying.

Children who are bullied are more likely to drop out of school, continuing to expand the educational divide and the continuation of chronic poverty. 
- $\quad$ They have a lower social, economic status, suffer from mental disorders, engage in risky behaviors, experience psychosis, and have a higher risk of having a substance use disorder.

- $\quad$ Bullied children have a difficult time keeping a job at the age of 24 to 26, and men who were bullied in high school had a significant chance of being unemployed at the age of 50 (Brimblecombe, Evans-Lacko, Knapp, King, Takizawa, Maughan, \& Arsenault, 2008).

Matthews, Jennings, Lee, \& Pardini (2017) discussed research showing that bullied children had a low SES, and they are less likely to have a high school diploma or GED. Throughout their lifetime, they have lower adult family income and lack adequate resources with lower social status in their community. Brimblecombe et al. (2008) reported that "four decades after the bullying occurred, both men and women who were bullied in childhood were less likely to be in employment and accumulated less wealth in the form of home-ownership or savings than participants who were not bullied" (p. 138). Research depicting those results can only strengthen chronic poverty with a new generation of people who will continue to reinforce and ensure generational poverty advances for the foreseeable future.

\section{TF-CBT}

The best method for treating bullying is prevention and having a zerotolerance in school and at home. McAdams and Schmidt (2007) purported that teachers have indicated feeling underprepared to recognize and manage bullies and bullying behaviors in their classroom. Their lack of knowledge causes them to ignore serious aggressive behaviors, or they identify bullying but feel inadequate or too afraid to intervene and address the problem. Parents and guardians are not equipped to train their children and convey the dangers of bullying, model a bully-proof home, and they lack the knowledge of teaching their children how to become resilient. Bullying that happens in the home is often overlooked by everyone involved because it is family-oriented. Bowes, Wolke, Joinson, Lereya, and Lewis (2014) proclaimed that sibling bullying is a specific type of aggressive behavior that is repeated over time (complex trauma) - intending to cause harm and dominate the weaker sibling. Studies show a strong correlation between sibling bullying and increased internalizing symptoms - resulting in the emergence of depression and self-harm in children, adolescent, and adulthood. It is essential to protect children in the two most important places where they spend a significant amount of timehome and school.

Children must become emotionally intelligent, develop coping skills, and use assertive communication (verbal and non-verbal) to deescalate and disengage from bullying behaviors and bullies. If a child is not safe within one's mind, he is not safe anywhere. The renewing of their mind must 
transform children by becoming bully-proof. Bullying survivors may have PTSD, and they do whatever it takes to escape the intrusive thoughts, shame, and humiliation of being demoralized. Steele and Malchiodi (2012) professed that "PTSD is more common in children than most physicians believe; therefore, the diagnosis can often be missed. Children being treated for behavioral problems may be suffering from PTSD" (p. 15). Black, Woodworth, Tremblay, and Carpenter (2012) proclaimed that "TF-CBT is a component-based model and can be summarized using the acronym "PRACTICE" (p. 196). Cognitive behavioral therapy (CBT) is an empirically supported approach to treating PTSD and CPTSD. According to Levers (2012), through the method, clients learn how to reduce negative emotional and behavioral responses that follow a traumatic event, because "the treatment is based, fundamentally, on learning and cognitive theories that address distorted beliefs and attributions related to the traumatic events experienced by an individual" (p. 496). As reported by Black et al. (2012),

In relation to treating trauma-related symptoms, there are a number of CBT-like treatments that involve most aspects of the therapy but do not actually present themselves specifically as CBT, such as multimodal trauma treatment (MMTT), as well as different variations of CBT, such as traumafocused cognitive behavioural therapy (TF-CBT). (p. 194)

TF-CBT is the treatment modality discussed in this paper as an efficient approach to treating bullying survivors suffering from symptoms of CPTSD. Johnson (2012) reported that trauma-focused care is done professionally and thoughtfully. It is informed of the overall construct showing great concern not only for the survivor's emotional safety but it is focused on the reassessment and restructuring of the treatment system to evaluate how the traumatic experience may affect the survivor's role and interaction with certain aspects of the entire systematic approach for treatment. One of the many benefits of TF-CBT is that multimodalities can be applied to reach the desired goals of hope, recovery, and resiliency, and the approach is substantiated by years of empirical and evidence-based research and advancing theories to wellness. As explained by Black et al. (2012),

During treatment, the traumatized child is provided with psychoeducation, taught relaxation skills, affective expression and modulation, and cognitive coping skills. In addition, they are encouraged to use trauma narration and cognitively process the trauma, use in vivo exposure to master trauma reminders, have conjoint parent-child sessions, and enhance safety and the trajectory of development. (p. 196)

Johnson (2012) stated that TF-CBT is the most studied of all the treatments for trauma-related symptoms among children and adolescents, even though many professionals do not recognize the advantages. Black et al. (2012) asserted that regardless of the specific treatment protocol that is 
adhered to, it is crucial to keep in mind that psychoeducation, coping skills, trauma narratives, cognitive restructuring, and creating an action plan for after the cessation of therapy are all typically integral practices of the traumainformed treatment programs. By implementing some or all of these critical practices, it should be possible to reduce trauma-related symptoms among trauma survivors. Even resilient children show distress following trauma exposure, and their reactions cause them to increase monitoring of their environment for potential dangers, experiencing anxiety when they are separated from trusted adults, or heightened need for affection, support, and reassurance because trauma re-enactments may resurface again in their lives (Kiser \& Black, 2005). Therefore, treating the psychological effects of bullying is critical to living a life free of chronic poverty. It is estimated that 160,000 school-aged children miss school because of bullies or bullying behaviors (Skaine, 2017). If children are too afraid or too humiliated to go to school, how will they ever learn the hidden rules of the middle-class and become educated, productive members of society?

\section{Chronic Poverty and the Educational Divide}

Individuals who live in poverty present a profound challenge in school systems, homes, and communities. Brito and Noble (2014) asserted that socioeconomically disadvantaged children tend to experience less linguistic, social, and cognitive stimulation from their caregivers and home environments than children from higher Socioeconomic Status (SES) homes" (p. 2). Hair, Hanson, Wolfe, and Pollak (2015) proclaimed that there is strong evidence that poverty influences language (temporal lobe) and executive functioning (frontal lobe). Deficits in the executive functioning of individuals in poverty have been found during the life course in studies conducted during infancy as well as in childhood, adolescence, and adulthood (p. 823). Mending the issues associated with chronic poverty and breaking generational curses is a massive undertaking, but several educational programs are intervening at an early age in the child's psychological and psychosocial development, but those initiatives alone do not alleviate the problems or lessen the psychological effects of poverty and trauma. Hair, Hanson, Wolfe, and Pollak (2015) reported that "children living in poverty have lower scores on standardized tests of academic achievement, more mediocre grades in school, and lower educational attainment (p. 823).

Parents must be taught and trained to become self-sufficient, improve self-esteem and self-compassion and learn to have high self-efficacy and the will and desire to change. Before tackling the trauma that is interwoven in poverty that causes a reoccurring cycle, society has to take an aggressive approach to improve the physical conditions, educate and train parents, and combat hunger and other lack of resources. Ruby Payne wrote a book entitle, 
A Framework for Understanding Poverty. According to Payne (1996), if people around a person has similar circumstances, and the problems attached to poverty, the notion of poverty and wealth is vague. Individuals bring the hidden rules of their environment, placing poverty-stricken communities at a disadvantage because schools and society operate from the middle-class norms and use the arcane rules of the middle class. The two most important conditions to help people move out of poverty are education and relationships (Payne, 1996).

How is it possible for parents to teach their children the hidden rules of common cultural patterns when all they know is the exposed rules of poverty? There must be a cultural shift to train parents to become knowledgeable and educated because education is the vehicle that will drive them away from poverty. The effects of generational poverty cannot be obliterated in an instance. It takes significant time to unravel generations of neural pathways that have been programmed to accept poverty. Society and educators must be willing to be patient, teach, and reprogram a culture of people by transforming their mental faculties. Amatea and West (2007) stated that rather than viewing poverty from society's perspective, inclusive of systemic influences and class privilege, many of these educators believe that poor people are intrinsically inferior because of their innate flaws such as lack of motivation or poor decision making. Parents and children living in poverty cannot be held accountable for their intrinsic defects. Until the generational narrative of poverty has been retold psychologically, spiritually, and emotionally, the problem will remain in today's societies. Poor people will always exist in the world; however, educators and society must be willing to lead, follow, or get out of the way, because if they are not ready to be part of the solution, their patronizing demeanor is part of the problem.

The educational gap between Blacks and Latinos and Whites has nothing to do with the intellectual abilities of the poverty-stricken minority population. In many minority families, getting a good education is not emphasized because survival is more important than learning. Children living in poverty do not receive support or challenges that are necessary to be successful in high school, college, and life. Since education is not essential to their parents, why should they make it vital for themselves? Some minority students overcome the odds and become successful; however, for the majority, these neglected students fall between the cracks.

There is a need to train parents on how to educate and instill the value of education in their children. van der Veen and Preece (2005) asserted that "the trend in adult education policies (where they exist at all) has been to focus on literacy or basic education. Research has shown that basic literacy skills are not in themselves sufficient to make a significant impact on poverty reduction, though they do help" (p. 381). Therefore, it would be beneficial to 
provide workshops, seminars, and hands-on training for parents of minorities living in poverty, but more is required in areas where a tremendous impact can be made to improve living conditions. van der Veen and Preece (2005) stated that targeting the real needs of the poor will take a collaborative approach between all stakeholders, employers, businesses, and employees. Poverty is an eyesore, and it does not benefit society, families, education, and the economy. Many parents do an excellent job rearing respectful citizens; however, they fail to train them to be self-sufficient, self-motivated, and educationally prepared to attend school, college, and productive members of society. When children are prepared at home, they come to school at an advantage, and they are eager to learn. A healthy family with solid values is the foundation for academic and personal success. Through adult education, what better way to model the importance of learning and to work to improve living conditions. The evidence on adult education in the public economic domain leads to the hypothesis that a "more extended and more targeted system for basic education, agricultural extension, and vocational training is urgently needed to help people to generate income" (van der Veen \& Preece, 2005, 390). If the root (family) is not nurtured and watered, the possibility for success and motivation will wither and die.

\section{Teacher's Perception and Stereotype Threats}

According to Dye (2014), research from previous studies revealed that teachers often doubt that education is vital for low-income students of color, and they have feelings of inadequacy when teaching minorities who live in poverty. The confident educator influences students' lives in ways unimaginable until many years later, when the former students share their experiences about that one instructor who made a difference. Many influential people contribute their success to a memorable teacher who encouraged them to be the best they could be in the classroom and beyond. Research has provided enough evidence to support that minorities do not learn the same way as their counterpart, and the same criterion does not motivate them. Educators must create culturally diverse environments, have belief in their pedagogical skills and abilities to connect and teach all students. Studies reported that educators could feel unequipped to teach culturally diverse students, reporting lower self-efficacy. Tucker, Porter, Reinke, Herman, Ivery, Mack, and Jackson (2005) postulated that a lack of efficacy could explain the educational divide between minorities living in poverty and the middle-class; however, school administrators must increase efforts to improve teacher efficacy because it is paramount to decrease the disproportionate high school dropout rates and underperforming children in the classroom.

When children are neglected, abused, and experience trauma, teachers are usually on the frontline, being a friend, mother-figure, and voice of reason. 
Teachers need to know their students' culture. According to Aldermann (2004), cultural competence helps educators to become astute to verbal and nonverbal cues from minorities, creating a classroom climate that encourages students to express themselves and feel accepted without losing their identity. School personnel, administrators, staff, and educators must see that there is a need to improvise and devise a plan to address the educational needs of culturally diverse students living in poverty. Cultural sensitivity is a process that will prevent stereotype threats in the classroom that decrease bias, prejudice, and demoralization that stifles the learning process.

Neuburger, Jansen, Heil, and Quaiser-Pohl (2012) purported that "stereotype threat is the event of a negative stereotype about a group to which one belongs becoming self-relevant and to being at risk of confirming, as selfcharacteristic, a negative stereotype about one's group" (p. 62). Stereotype threat can disrupt educational performance, causing low self-efficacy, low self-esteem, and a significant decrease in academic motivation. Studies show that the threat of stereotype causes minorities to not only struggle academically, but it asphyxiates personal growth and social development. Stereotype threat is indoctrinated in the belief that anyone in a stereotypical group can feel pressure to avoid fulfilling negative expectations. The pressure of becoming a self-fulfilling prophecy bolsters underperformance, which is the most detrimental barrier to achievement. There is no doubt that minorities have equal potential to perform as well as their counterpart; however, when minority students know that they are being compared, they tend to perform discouragingly.

As reported by Neuburger et al. (2012), stereotype threat debilitates performance because it initiates three different mechanisms that reduce the capacity of working memory (physiological stress, monitoring processes, and emotion-suppressing efforts). The mechanisms evoke a cognitive imbalance between the person's concept of self, ability domain, and group. Stereotype threat accentuates minorities perception of being inferior; therefore, they take on an inferiority complex of their own, contributing to their lack of confidence in their abilities, accepting the façade that white students are smarter than they and educators view them as inept. The research does not support that stereotype threats occur in white teachers only but any educator who feels a particular population, such as those living in poverty, is beneath them and they hold poverty-stricken people responsible for a life of deprivation. Amatea and West (2007) proclaimed that Many educators view poor people as morally and culturally deficient. Believing that poor families have attitudes, values, and behaviors that sustain their position at the bottom of the economic ladder, these educators often blame parents for passing on these traits to their children instead of transmitting the middle-class cultural patterns they believe are necessary to succeed in school and in life. (p. 83) 
Research supports that students who can identify with their academics and self-esteem have a better chance of being successful. Minority students try to accomplish goals and achieve a sound education as everyone else, but chronic poverty and teacher's perception cause them to struggle and fall behind. To succeed, minorities living in poverty are trying too hard because they are dealing with the threat of stereotype and disidentification. It could be assumed that hard work, dedication, and commitment to educational endeavors would close the gap and help minorities excel; however, trying harder adds an extra burden, causing more problems such as higher levels of stress, anxiety, and depression. What options are left for minority children living in poverty?

\section{Implications and Conclusion}

Living a life of chronic poverty that increases the risk of trauma, victimization, bullying and its psychological effects, becoming a high school dropout, and being educated by a system that was not designed for people living in deprivation is not a situation that anyone would choose. Trauma plays a significant role in the lives of people living in poverty. Traumatic experiences are subjective, but the concept of trauma suggests that the wounded is confronted by an enormous life-changing event, such as divorce, death of a loved one, unemployment or terminated, diagnosis of illness or disease, or culture shock; however, trauma is generally referred to a severe or psychological injury sustained as a result of a life-threatening or horrific experience (Stebnicki, 2017). Bullying is a significant problem for children, and the epidemic warrants the attention of policymakers, educators, parents, church leaders, and community stakeholders. Society is doing a poor job of protecting and educating children. Vanderbilt and Augustyn (2010) discussed the significance of people being more vigilant and aggressive by not tolerating bullying and modeling that it is not welcomed in the home, community, and school. Particular focus must be placed on children or adolescents who have chronic medical illnesses like acne, being medically obsessed, severe eczema, physical deformities like cleft lip and palate, or some who suffer from neuropsychological disorders like learning disabilities, down syndrome, and autism spectrum disorder, making them potential targets for bullying and demoralization. School bullying is a widespread phenomenon not only in the United States of America but in many countries around the world, signifying that bullying should be considered a significant international public health problem (Collier, 2013).

Research proclaims that today's school counselors can bring unique skills to help educators working with students in high-poverty schools who experience bullying and the psychological effects. Working with this population provides a significant opportunity for school counselors to broaden 
their role with educators, staff, office personnel, and administrators beyond only delivering services to individual students but taking a collaborative approach (Amatea \& West, 2007). Poverty-stricken minorities can accomplish great things academically when teachers and others in the learning environment create an atmosphere that makes them feel confident. Children can learn and achieve at very high levels, once they gain confidence in their abilities and accept that their thinking and efforts can help them do well. It takes a cumulative effort to make a substantial change in the classroom, and everyone must accept his or her role. Amatea and West (2007) declared that

These roles are (a) serving as a cultural bridge between teachers and students and blocking the blaming that often derails efforts to work with poor students and their families, (b) functioning as a pedagogical partner with teachers by connecting the curriculum more directly to students' lives, and (c) teaming with teachers to create a more welcoming, family-centric school climate. (p. 82)

School systems and administrators must make the cultural transformation a priority and not an afterthought or placed at the bottom of the school's agenda. Neuburger et al. (2012) declared that stereotype threat and self-fulfilling prophecies play an instrumental role in socialization and are part of the hidden curriculum in schools. Adding the hidden curriculum to the hidden rules of the middle-class, minorities living in poverty do not have a fighting chance and will remain hidden in the classroom to continue the cycle of generational poverty. Head Start, 2 Gen Programs, Babies "Can't" Wait, and other programs have been established to address the disparity in the vulnerable population; however, a tremendous amount of work must be put into action along with robust educational reforms.

No Child Left Behind (NCLB) was designed to ensure that all schools meet the same academic standards and achievement levels, regardless of the school system was in an area where poverty is high or not. Research proves that the NCLB has been nothing but an ultimate failure because state and federal standards cannot establish guidelines on the hidden rules of education that was designed for the middle-class. Ladd (2012) reported that under NCLB, "each school must meet the same standard, regardless of whether it serves low- or high-SES students and must do so for all relevant subgroups within the school defined by income, minority status, and Limited English Proficient status" (p. 213). Poverty-stricken children will always be a general population of people in the middle-class classroom, skewing achievement expectations and standards. What must happen for educators to improve their low self-efficacy and lack of confidence to teach children from lower SES?

Teachers must become culturally competent, demonstrating relevant pedagogy techniques that encourage educators to understand local students, cultures, demographics, and the hidden rules of poverty. It is equally crucial 
for middle-class teachers to understand children and parents living in deprivation as it is for people living in poverty to learn the hidden rules of the middle-class. Matsko and Hammerness (2014) suggested that multicultural education create dialogue and relevancy to bridge the gap between educators, students, state and federal standards, and the school's curriculum. The faculty must understand the significance of the teacher-student relationship, their respective cultures, and how the dynamics of education are interwoven in the classroom. Teachers do not have to go to an elite college or university to reach, teach, and empower children living in poverty. They must be willing to accept the fact that these children will always be their classrooms, become flexible and willing to change to become the best educator for all students and take those teachable moments to learn more about those students who come from a different geographical area. Mattsko and Hammerness (2014) cited that educators must demonstrate how "context extends well beyond one's immediate physical surroundings, and in doing so, we illustrate how a simple understanding of context can be expanded to include state and federal policy, the neighborhood, the district, and urban public school classroom" (p. 137).

This burden of educating the underserved does not fall solely on the educational system, but the parents must take a proactive approach to improve their education and the education of their children. Education starts at home with family and community. Families must provide stability, the foundation for education, and relay the importance of learning to their children. When a family gives their children support, challenges, and stimulate their children, minorities are more likely to choose harder subjects in high school, get better grades, end up in better colleges, and have higher self-esteem in college and beyond. Challenges empower minority children by giving them a vision, direction, focus, and perseverance to succeed. A new approach called 2 Gen helps families break the cycle of poverty by simultaneously addressing the needs of parents and children to improve significant outcomes for the entire family ("Two-Generation Approaches," 2018). Familial support provides the solace that allows freedom and closes the door on stress, worries, and fears. Parents living in poverty must become educated and learn to be their children's first teacher. According to "Two-Generation Approaches" (2018), "Twogeneration strategies can be used in many policy areas, including human services, education, labor and workforce, and health. They can be used to address a myriad of policy issues, including poverty, literacy, school readiness, and family economic stability" (p. 3).

The research presented here does not touch the surface of what it will take to disassociate poverty from bullying, the disproportionate rate of traumatic experiences, and the complicated process of closing the educational divide by educating all students regardless of low socioeconomic status, demographics, cultures, races, and ethnicities of the underserved, vulnerable 
population. Payne (1996) purported that "support systems need to include the teaching of procedural self-talk, positive self-talk, planning, goal-setting, coping strategies, appropriate relationships, options during problem-solving, access to information and know-how, and connections to additional resources" (p. 75). To make changes and see significant differences, society must be willing to challenge the status quo and change the culture by breaking the cycle of generational poverty - equipping people living below the poverty level to use education as the catalyst to move from poverty to middle-class - where the hidden rules to function in society are available for everyone.

The United States is an individualistic society, and too many people are only concerned about their small microcosm. It is going to take a collectivist approach to stomp out the harmful effects of bullying and its detrimental ramifications later in life. Bullying is not a rite of passage, and if not treated, they will carry the trauma and hidden scars into their adult life. Society must stop considering bullying as a school ritual but recognize it as a public health crisis. Skaine (2015) reported that 19 percent of U.S. elementary students are bullied, and more than 160,000 kids stay home from school because they are afraid as published by Vanderbilt and Augustyn (2010), bullying, whether as bullies, victims and bully-victims, is correlated with poorer outcomes in the classroom and society. Bullying involvement is significant because it can lead to poor psychosocial adjustment, greater health problems, poverty, and deficient emotional and social adjustment (p. 316). Bullying behaviors and their lasting effects have the propensity to continue the cycle of chronic poverty, so the generational curse continues with no end if a cultural shift does not happen soon. People who live in poverty experience higher rates of trauma and bullying. Children who are bullied are more likely to drop out of school, have a lower social, economic status, suffer from mental disorders, engage in risky behaviors, and substance use disorders. According to Matthews, Jennings, Lee, \& Pardini (2017), research showed that bullied children had a low SES, and they are less likely to have a high school diploma or GED. Throughout their lifetime, they have lower adult family income and lack adequate resources with lower social status in their community. Studies depicting those results strengthen chronic poverty with a new generation of people contributing to the poverty-stricken life cycle.

Taylor and Barrett (2018) professed that raising awareness of early trauma is a critical first step to reduce its impact, but most of the contemporary literature focuses on the actual incidence of trauma. As denoted, trauma is not necessarily an isolated incident but the culmination of traumatic events and episodes in the contextual environment. Society must construct a plan that addresses the mental, physical, psychological, and cognitive faculties of all individuals living in poverty. 2 Gen is very promising because it focuses on educating the parents, children, and community by working from the inside 
out. According to Taylor and Barrett (2018), educators cannot prevent traumatic experiences from occurring; however, they can seek to mitigate their impact by implementing a range of approaches and interventions such as R4L," Readiness for Learning (R4L), a 'Brain-based, Attachment-Led, Trauma-Informed Community intervention' (BALTIC) approach to supporting learners to be settled and ready to learn" (p. 65). Collected data indicates that R4L has made significant progress in the classroom with a proven increase in students concerning self-regulation and executive functioning (Taylor \& Barret, 2018). In summation, Ladd (2012) reported that policymakers must assure that children attending schools that educate large proportions of disadvantaged children must provide them access to highquality teachers, principals, supports for students, and other resources, holding school systems accountable for the quality of education provided and their internal processes and practices.

\section{References:}

1. Alderman, M. K. (2004). Motivation for achievement: Possibilities for teaching and learning $\left(2^{\text {nd }} e d\right)$. Mahwah, NJ: Lawrence Erlbaum Associates.

2. Amatea, E. S., \& West-Olatunji, C. (2007). Joining the conversation about educating our poorest children: Emerging leadership roles for school counselors in high-poverty schools. Professional School Counseling, 11(2), 81-89.

3. Bilic, V. (2015). The role of socioeconomic differences and material deprivation in peer violence. Epiphany, 8(2), 177-202. doi:http://dx.doi.org/10.21533/epiphany.v8i2.169.g137

4. Black, P. J., Woodworth, M., Tremblay, M., \& Carpenter, T. (2012). A review of trauma-informed treatment for adolescents. Canadian Psychology, 53(3), 192-203.

5. Blad, E. (2016). Bullying: Preventing bullying through science, policy, and practice. Education Week, 35(31)

6. Blair, C., \& Raver, C. C. (2016). Poverty, stress, and brain development: New directions for prevention and intervention. Academic pediatrics, $16(3 \quad$ Suppl), S30-S36. doi:10.1016/j.acap.2016.01.010

7. Bowes, L., Wolke, D., Joinson, C., Lereya, S. T., \& Lewis, G. (2014). Sibling bullying and risk of depression, anxiety, and self-harm: A prospective cohort study. Pediatrics, 134(4). doi:10.1542/peds.20140832

8. Brimblecombe, N., Evans-Lacko, S., Knapp, M., King, D., Takizawa, R., Maughan, B., \& Arseneault, L. (2018). Long term economic impact associated with childhood bullying victimisation. Social Science and 
Medicine, 208

$134-141$ doi:https://doi.org/10.1016/j.socscimed.2018.05.014

9. Brito, N. H., \& Noble, K. G. (2014). Socioeconomic status and structural brain development. Frontiers in Neuroscience, 8, 276. doi:10.3389/fnins.2014.00276

10. Briere, J. N., \& Scott, C. (2015). Principles of trauma therapy: A guide to symptoms, evaluation, and treatment (2nd ed., p. 10). Thousand Oaks, CA: Sage Publications.

11. Center for Substance Abuse Treatment (US). Trauma-informed care in behavioral health services. Rockville (MD): Substance Abuse and Mental Health Services Administration (US); 2014. (Treatment Improvement Protocol (TIP) Series, No. 57.) Chapter 2, Trauma Awareness. Available from: https://www.ncbi.nlm.nih.gov/books/NBK207203/

12. Collier, R. (2013). Bullying symptoms. Canadian Medical Association Journal, 85(16), E754. doi.10.1503/cmaj.109-4610

13. Collins, K., Connors, K., Donohue, A., Gardner, S., Goldblatt, E., Hayward, A., Kiser, L., Strieder, F. Thompson, E. (2010). Understanding the impact of trauma and urban poverty on family systems: Risks, resilience, and interventions. Baltimore, MD: Family Informed Trauma Treatment. http://nctsn.org/nccts/nav.do?pid=ctr_rsch_prod_ar or http://fittcenter.umaryland.edu/WhitePaper.aspx

14. Dye, L. (2014). School counselors' activities in predominantly African American schools: an exploratory study. Michigan Journal of Counseling, 41(1), 18-37. doi:10.22237/mijoc/1393632120

15. Evans, G. W., \& Kim, P. (2012). Childhood poverty, chronic stress, self-regulation, and coping. Child Development Perspective, 7(1), 4348. doi:https://doiorg.ezproxy.liberty.edu/10.1111/cdep.12013

16. Gabrielli, J., Gill, M., Koester, L. S. and Borntrager, C. (2014). Psychological perspectives on 'acute on chronic' trauma in children: Implications of the 2010 earthquake in Haiti. Child \& Society, 28: 438450. doi:10.1111/chso. 12010

17. Gonzalez-Gonzalez, N. L., Suarez, M. N., Perez-Pinero, B., Armas, H., Domenech, E. M., \& Bartha, J. L. (2006). Persistence of fetal memory into neonatal life. Acta Obstetricia et Gynecologica Scandinavica, 85(10), 1160-1164. doi:https://doi.org/10.1080/00016340600855854

18. Hair, N. L., Hanson, J. L., Wolfe, B. L., \& Pollak, S. D. (2015). Association of child poverty, brain development, and academic achievement. JAMA Pediatrics, 169(9), 822-829. doi:10.1001/jamapediatrics.2015.1475 
19. Hatcher, A. M., Gibbs, A., Jewkes, R., McBride, R., Peacock, D., \& Christofides, N. (2019). Effect of childhood poverty and trauma on adult depressive symptoms among young men in Peri-Urban South African settlements. Journal of Adolescent Health, 64(1), 79-85. doi:https://doi.org/10.1016/j.jadohealth.2018.07.026

20. Hendricks, E. A., \& Tanga, P. T. (2019). Effects of bullying on the psychological functioning of victims. Southern African Journal of Social Work \& Social Development, 31(1), 1-17. https://doiorg.ezproxy.liberty.edu/10.25159/2415-5829/3939

21. Herman, J. (2001). Trauma and recovery: The aftermath of violencefrom domestic violence to political terror. New York, NY: Basic Books.

22. Herman, J. (2012). CPTSD is a distinct entity: Comment on Resick et al. (2012). Journal of Traumatic Stress, 25, 256-257. doi:10.1002/jts.21697

23. James, D. K. (2010). Fetal learning: a critical review. Infant \& Child Development, 19(1), 45-54. https://doiorg.ezproxy.liberty.edu/10.1002/icd.653

24. Johnson, B. C. (2012). Aftercare for survivors of human trafficking. Social Work and Christianity, 39(4), 370-389.

25. Kim, S. Fonagy, P., Allen, J., \& Strathearn, L. (2014). Mothers' unresolved trauma blunts amygdala response to infant distress, Social Neuroscience, 9:4, 352-363. 10.1080/17470919.2014.896287

26. Kiser, L. J., \& Black, M. M. (2005). Family processes in the midst of urban poverty: What does the trauma literature tell us? Aggression and Violent Behavior, 10(6), 715-750. doi:https://doi.org/10.1016/j.avb.2005.02.003

27. Klest, B. (2012). Childhood trauma, poverty, and adult victimization. Psychological Trauma: Theory, Research, Practice, and Policy, 4(3), 245-251. http://dx.doi.org.ezproxy.liberty.edu/10.1037/a0024468

28. Ladd, H. F. (2012). Education and poverty: Confronting the evidence. Journal of Policy Analysis and Management, 31(2) 203-227. doi:10.1002/pam.21615

29. Levers, L. L. (2012). Trauma counseling: Theories and interventions (p. 496). New York, NY: Springer Publishing Company.

30. Lowdermilk, E., \& Brunache, J. (2013). Experiencing unexpected pathways: A grounded theory study of the surprising transformation of inner-city youth. Social Work and Christianity, 40(3), 322-351.

31. Matsko, K. K., \& Hammerness, K. (2014). Unpacking the "urban" in urban teacher education: Making a case for context-specific preparation. Journal of Teacher Education, 65(2), 128-144. doi: $10.1177 / 0022487113511645$ 
32. Matthews, K. A., Jennings, J. R., Lee, L., \& Pardini, D. A. (2017). Bullying and being bullied in childhood are associated with different psychosocial risk factors for poor physical health in men. Association for Psychological Science, 28(6), 808-821. doi:10.1177/0956797617697700

33. McAdams, C. R., \& Schmidt, C. D. (2007). How to help a bully: Recommendations for counseling the proactive aggressor. Professional School Counseling, 11(2), 120-128. Retrieved from https://www.schoolcounselor.org/asca/media/asca/home/McAdamsV ol11No2-(1).pdf

34. Myers, H. F., Wyatt, G. E., Ullman, J. B., Loeb, T. B., Chin, D., Prause, N., . . . Liu, H. (2015). Cumulative burden of lifetime adversities: Trauma and mental health in low-SES African Americans and Latino/as. Psychological Trauma: Theory, Research, Practice, and Policy, $7(3)$, 243-251. http://dx.doi.org.ezproxy.liberty.edu/10.1037/a0039077

35. Neuburger, S., Jansen, P., Heil, M., \& Quaiser-Pohl, C. (2012). A threat in the classroom: Gender stereotype activation and mentalrotation performance in elementary-school children. Zeitschrift fur Psychologie, 220(2), 61-69. doi:10.1027/2151-2604/a000097

36. Patrick, P. D., Lipina, Colombo, S. J., \& Jorge, A. (2010). Poverty and brain development during childhood: An approach from cognitive psychology and neuroscience. Journal of the International Neuropsychological Society, 16(3), 582-584. doi:http://dx.doi.org.ezproxy.liberty.edu/10.1017/S135561771000039 1

37. Payne, R. K. (1996). A framework for understanding poverty (4th ed., p. 75). Highlands, TX: aha! Process, Inc.

38. Shamai, M. (2018). Is poverty a collective trauma? A joint learning process with women living in poverty in the city of Haifa in Israel. The British Journal of Social Work, 48(6), 1718-1735. https://doi.org/10.1093/bjsw/bcx116

39. Skaine, R. (2015). Abuse: An encyclopedia of causes, consequences, and treatments (p. 40). Santa Barbara, CA: Greenwood.

40. Stebnicki, M. A. (2017). Disaster mental health counseling: Responding to trauma in a multicultural context. New York, NY: Springer Publishing Company.

41. Steele, W., \& Malchiodi, C. A. (2012). Trauma-Informed practices with children and Adolescents (p. 15). New York, NY: Routledge.

42. Stewart, P. (2014). Suffering fiction in the unnamable. Samuel Beckett Today/ Aujourd'Hui, 26, 165-177,315. 
43. Tariq, Q., \& Tayyab, A. (2011). Bullying and its psychological effects on adult and adolescent students. Journal of Educational Research, 14(2), 22-31.

44. Taylor, L., \& Barrett, W. (2018). Developing a trauma-informed approach to closing the poverty-related attainment gap. Educational and Child Psychology, 35(3), 64-75.

45. Tucker, C. M., Porter, T., Reinke, W. M., Herman, K. C., Ivery, P. D., Mack, C. E., \&Jackson, E. S. (2005). Promoting teacher efficacy for working with culturally diverse students. Preventing School Failure, $50(1)$, 29-34. doi:https://doiorg.ezproxy.liberty.edu/10.3200/PSFL.50.1

46. Two-Generation approaches to addressing poverty: A toolkit for state legislators (2018). In National Conference of State Legislatures, (p. 3).

47. Uhernik, J. A. (2017). Using Neuroscience in trauma therapy, Creative and compassionate counseling (p. 54) New York, NY: Routledge.

48. Vanderbilt, D., \& Augustyn, M. (2010). The effects of bullying. Paediatrics and Child Health, 20(7), 315-320. doi:https://doi.org/10.1016/j.paed.2010.03.008

49. van der Veen, R., \& Preece, J. (2005) Poverty reduction and adult education: Beyond basic education, International Journal of Lifelong Education, 24(5), 381-391. doi: 10.1080/02601370500169723

50. Wagner, K. D. (2016, November 29). Effects of childhood trauma on depression and suicidality in adulthood. Psychiatric Times, 33(11) 